\title{
Surface-Doped Quasi-2D Chiral Organic Single Crystals for Chiroptical Sensing
}

Xiaobo Shang, ${ }^{\dagger},{ }^{\|, \perp}$ Inho Song, ${ }^{\dagger, \perp}$ Jeong Hyeon Lee, ${ }^{\ddagger}$ Wanuk Choi, ${ }^{\S}$ Jaeyong Ahn, ${ }^{\dagger}$ Hiroyoshi Ohtsu, ${ }^{\#}$ Jin Chul Kim, ${ }^{\ddagger}$ Jin Young Koo, ${ }^{\Delta}$ Sang Kyu Kwak, ${ }^{*}, \dot{\ddagger}$ and Joon Hak Oh${ }^{*}, \dagger$

${ }^{\dagger}$ School of Chemical and Biological Engineering, Institute of Chemical Processes, Seoul National University, 1 Gwanak-ro, Gwanak-gu, Seoul 08826, Republic of Korea

E-mail: joonhoh@snu.ac.kr

" Chemistry Research Laboratory, Department of Chemistry, University of Oxford, Mansfield Road, Oxford OX1 3TA, UK

$\$$ Department of Energy Engineering, School of Energy and Chemical Engineering, Ulsan National Institute of Science and Technology (UNIST), Ulsan 44919, Republic of Korea E-mail: skkwak@unist.ac.kr

${ }^{\S}$ Energy Materials Laboratory, Korea Institute of Energy Research (KIER), Daejeon, 34129, Republic of Korea

\# Department of Chemistry, School of Science, Tokyo Institute of Technology, 2-12-1 Ookayama, Meguro-ku, Tokyo 152-8550, Japan

${ }^{\Delta}$ Department of Chemistry, Pohang University of Science and Technology (POSTECH), Pohang, Gyeongbuk 37673, Republic of Korea

Keywords: supramolecular chirality, quasi-2D organic single crystals, optoelectronics, circularly polarized light detection, hydrazine doping 


\section{Supporting Contents}

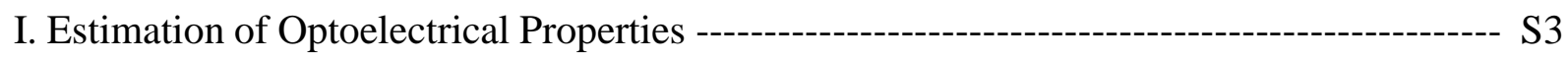

II. X-ray Crystallography ------------------------------------------------------------------------------ S4

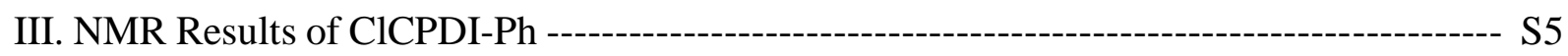

\section{Supporting Figures}

Crystal structures of ClCPDI-Ph-DMF single crystals (Figure S1) ----------------------------- S7

Optical images of ClCPDI-Ph-CF single crystals (Figure S2) ------------- S7

Optical images of ClCPDI-Ph-DMF single crystals (Figure S3) --------------------------------- S8

SAED patterns of single crystals obtained from TEM analysis (Figure S4) -------------------- S8

Self-assembly trend of ClCPDI-Ph-DMF crystals (Figure S5) ----------------- S9

Cyclic voltammetric measurement results of ClCPDI-Ph solution (Figure S6) ---------------- S9

Quantum calculation result of ClCPDI-Ph monomer using DFT method (Figure S7) ------- S10

Electrical properties of $(R)$-ClCPDI-Ph-DMF single crystals OFETs (Figure S8) ------------ S10

Electrical properties of ClCPDI-Ph-CF single crystals OFETs depending on the hydrazine doping time (Figure S9) -------------------------------------------------------------- S11

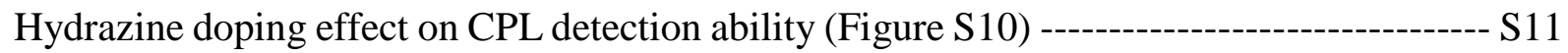

ESR spectra of $(R)$-ClCPDI-Ph-CF single crystals (Figure S11) ---------------------------- S12

UV-vis spectra of $(R)$-ClCPDI-Ph-CF single crystals before and after hydrazine doping --- S12 (Figure S12)

Molecular orbitals of two stacked $(R)$-ClCPDI-Ph from DFT calculations (Figure S13) ---- S13

\section{Supporting Tables}

Energy level of enantiomeric ClCPDI-Ph from CV measurement and DFT calculation ----- S14 (Table S1)

Optoelectronic characteristics of $(R)-\mathrm{Cl}$-CPDI-Ph-CF single crystals (Table S2) ------------ S14

Doping effect of hydrazine on electron affinity of $(R)$-ClCPDI-Ph molecule (Table S3) ---- S14 


\section{Estimation of Optoelectrical Properties}

In order to investigate photosensitivity for OPTs, photoresponsivity $(R)$ and photocurrent/dark-current ratio $(P)$ were calculated from transfer characteristics coupled with light irradiation. The $R$ and $P$ values are typically defined by the following equations:

$$
\begin{aligned}
& R=\frac{I_{\mathrm{ph}}}{P_{\mathrm{inc}}}=\frac{I_{\text {light }}-I_{\mathrm{dark}}}{P_{\mathrm{inc}}} \\
& P=\frac{I_{\text {light }}-I_{\mathrm{dark}}}{I_{\mathrm{dark}}}
\end{aligned}
$$

where $I_{\mathrm{ph}}$ is the photocurrent, $P_{\text {inc }}$ the incident illumination power on the channel of the device, $I_{\text {light }}$ the drain current under illumination, and $I_{\text {dark }}$ the drain current in the dark, respectively. In addition, the external quantum efficiency (EQE) $(\eta)$ of OPTs was calculated which can be defined as the ratio of number of photogenerated carriers that practically enhances the drain current to the number of photons incident onto the OPT channel area, using the following equation $(\mathrm{S} 3)$ :

$$
\eta=\frac{\left(I_{\text {light }}-I_{\text {dark }}\right) h c}{e P_{\text {int }} A \lambda_{\text {peak }}}
$$

where $h$ is the plank constant, $c$ the speed of light, $e$ the fundamental unit of charge, $A$ the area of the transistor channel, and $\lambda_{\text {peak }}$ the peak wavelength of the incident light, respectively. Detectivity usually describes the smallest detectable signal, which allows comparisons of photodetector devices with different configurations and areas. $D^{*}$ was evaluated within this study using the following Equations (S4) and (S5):

$$
\begin{aligned}
& D^{*}=\frac{\sqrt{A}}{N E P} \\
& N E P=\frac{\sqrt{\overline{I_{n}^{2}}}}{R}
\end{aligned}
$$

In these equations, $A$ is the photodetector active area, NEP the noise equivalent power, and $\overline{I_{n}^{2}}$ the measured noise current. If the major limit to detectivity is shot noise from the drain current under dark conditions, $D^{*}$ can be simplified as:

$$
D^{*}=\frac{R}{\sqrt{\left(2 e \cdot I_{\text {dark }} / A\right)}}
$$




\section{X-ray Crystallography}

\section{X-ray Crystallographic Data for $(S)$-CICPDI-Ph-CF Single Crystal}

$\mathrm{C}_{41} \mathrm{H}_{23} \mathrm{Cl}_{6.75} \mathrm{~N}_{2} \mathrm{O}_{4} M_{r}=846.90$, crystal dimensions $0.40 \times 0.20 \times 0.03 \mathrm{~mm}^{3}$, monoclinic, space group $P 2_{1}, a=11.486(2) \AA, b=30.043(6) \AA, c=11.821(2) \AA, \beta=118.92(3)^{\circ}, V=3570.4(15)$ $\AA^{3}, Z=4, \rho_{\text {calcd }}=1.576 \mathrm{~g} \mathrm{~cm}^{-3}, \mu=0.422 \mathrm{~mm}^{-1}, \lambda=0.6600 \AA$ (synchrotron), $T=100(2) \mathrm{K}$, 21501 reflections out of 22678 with $I>2 \sigma(I), 976$ parameters, 931 restraints, $1.828<\theta<$ $29.035^{\circ}$, final $R$ factors $R 1=0.0433$ and $\mathrm{w} R 2=0.1136, \mathrm{GOF}=1.044$. Flack parameter: $0.00(4)$. CCDC : 1563122

\section{X-ray Crystallographic Data for (S)-CICPDI-Ph-DMF Single Crystal}

$\mathrm{C}_{42.67} \mathrm{H}_{28.17} \mathrm{Cl}_{4} \mathrm{~N}_{2.83} \mathrm{O}_{4.83} M_{r}=799.65$, crystal dimensions $1.00 \times 1.00 \times 0.30 \mathrm{~mm}^{3}$, trigonal, space group $P 3_{2}, a=11.8747(17) \AA, c=43.884(9) \AA, V=5359.0(19) \AA^{3}, Z=6, \rho_{\text {calcd }}=1.487$ $\mathrm{g} \mathrm{cm}^{-3}, \mu=0.269 \mathrm{~mm}^{-1}, \lambda=0.6600 \AA$ (synchrotron), $T=100(2) \mathrm{K}, 16165$ reflections out of 20994 with $I>2 \sigma(I), 994$ parameters, 1 restraints, $1.839<\theta<28.128^{\circ}$, final $R$ factors $R 1=$ 0.0688 and $\mathrm{w} R 2=0.1682, \mathrm{GOF}=0.994$. Flack parameter: $0.01(6) . \mathrm{CCDC}: 1563121$

\section{X-ray Crystallographic Data for $(R)$-CICPDI-Ph-CF Single Crystal}

$\mathrm{C}_{41} \mathrm{H}_{23} \mathrm{Cl}_{6.75} \mathrm{~N}_{2} \mathrm{O}_{4} M_{r}=846.90$, crystal dimensions $0.40 \times 0.20 \times 0.03 \mathrm{~mm}^{3}$, monoclinic, space group $P 2{ }_{1}, a=11.706(6) \AA, b=30.339(15) \AA, c=12.025(6) \AA, \beta=118.785(14){ }^{\circ}, V=$ 118.785(14) $\AA^{3}, Z=4, \rho_{\text {calcd }}=1.503 \mathrm{~g} \mathrm{~cm}^{-3}, \mu=0.559 \mathrm{~mm}^{-1}, \lambda=0.71073 \AA$ (Mo $\left.K \alpha\right), T=$ $100(2) \mathrm{K}, 10730$ reflections out of 17195 with $I>2 \sigma(I), 980$ parameters, 741 restraints, 1.342 $<\theta<27.484^{\circ}$, final $R$ factors $R 1=0.0685$ and $\mathrm{w} R 2=0.1735, \mathrm{GOF}=0.996$. Flack parameter: $-0.01(9)$. CCDC : 1562171

\section{X-ray Crystallographic Data for (R)-CICPDI-Ph-DMF Single Crystal}

$\mathrm{C}_{42.5} \mathrm{H}_{27.83} \mathrm{Cl}_{4} \mathrm{~N}_{2.83} \mathrm{O}_{4.83} M_{r}=797.31$, crystal dimensions $0.20 \times 0.20 \times 0.03 \mathrm{~mm}^{3}$, trigonal, space group $P 3_{1}, a=11.8350(17) \AA, c=43.729(9) \AA, V=5304.4(19) \AA^{3}, Z=6, \rho_{\text {calcd }}=1.498 \mathrm{~g} \mathrm{~cm}^{-}$ ${ }^{3}, \mu=0.388 \mathrm{~mm}^{-1}, \lambda=0.7000 \AA$ (synchrotron), $T=100(2) \mathrm{K}, 12836$ reflections out of 14253 with $I>2 \sigma(I), 993$ parameters, 1 restraints, $1.957<\theta<25.944^{\circ}$, final $R$ factors $R 1=0.0861$ and $\mathrm{w} R 2=0.2344, \mathrm{GOF}=1.044$. Flack parameter: 0.01(3). CCDC $: 1562170$ 


\section{NMR Results of CICPDI-Ph}

a. $(R)$-CICPDI-Ph

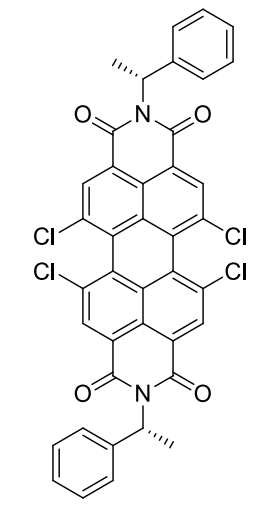

$(R)$-ClCPDI-Ph
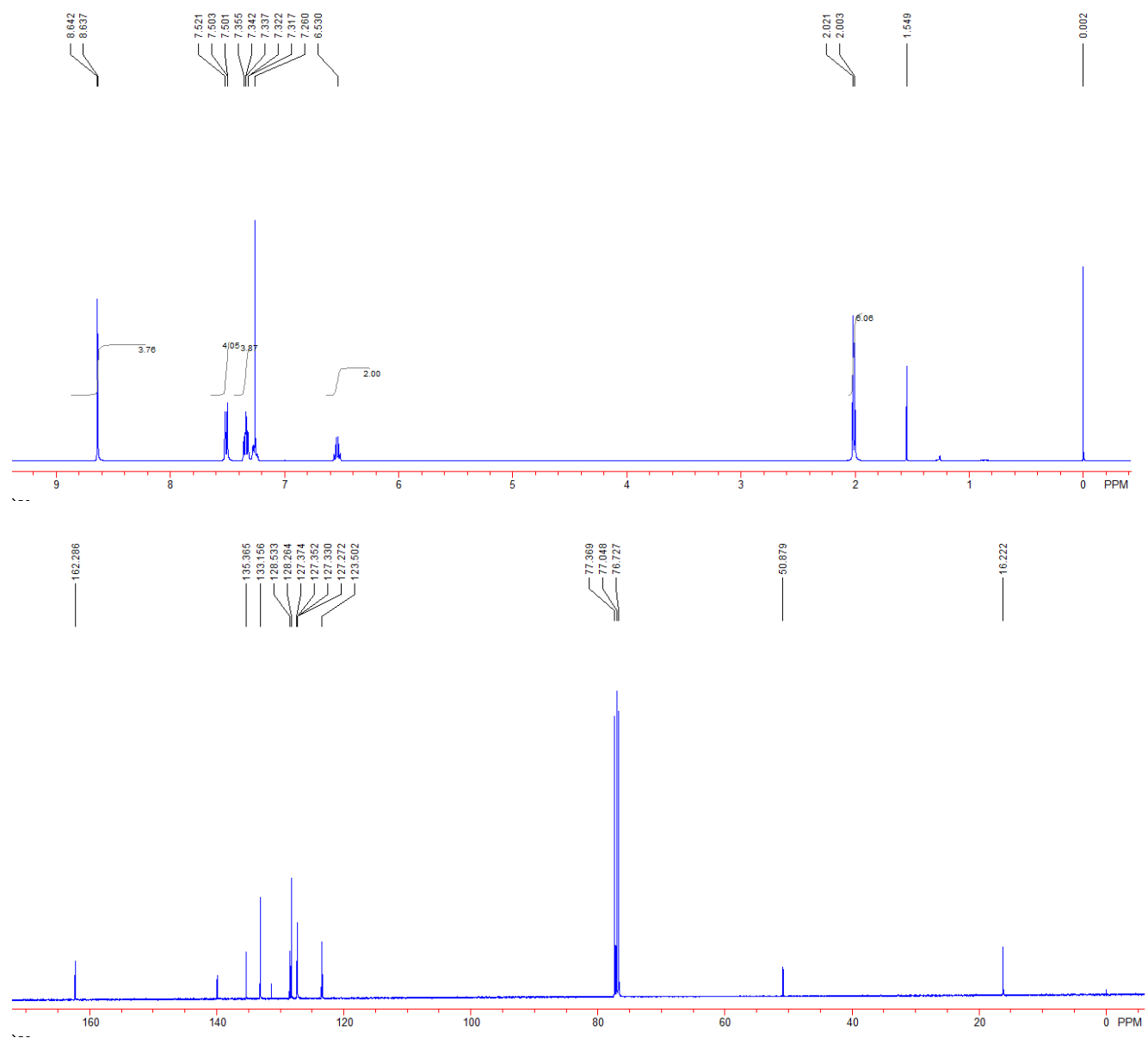

${ }^{1} \mathrm{H}$ NMR (400 MHz, $\mathrm{CDCl}_{3}$ ): $\delta 8.64$ (s, $\left.2 \mathrm{H}\right), 8.64$ (s, $\left.2 \mathrm{H}\right)$, 7.49-7.53 (m, $\left.4 \mathrm{H}\right)$, 7.30-7.37 (m, 4 $\mathrm{H}), 7.22-7.30(\mathrm{~m}, 2 \mathrm{H}), 6.54(\mathrm{q}, J=7.6 \mathrm{~Hz}, 2 \mathrm{H}), 2.01(\mathrm{~d}, J=7.6 \mathrm{~Hz}, 6 \mathrm{H}) \mathrm{ppm} .{ }^{13} \mathrm{C}$ NMR $(100$ $\left.\mathrm{MHz}, \mathrm{CDCl}_{3}\right): \delta 162.3,139.9,135.4,133.2,131.4,128.5,128.3,127.4,127.4,127.3,127.3$, 123.5, 123.4, 50.9, $16.2 \mathrm{ppm}$. HRMS $(\mathrm{m} / \mathrm{z})$ : $\left[\mathrm{M}^{+}\right]$calcd for 734.0334, found: 735.0415 . 
b. $(S)$-CICPDI-Ph

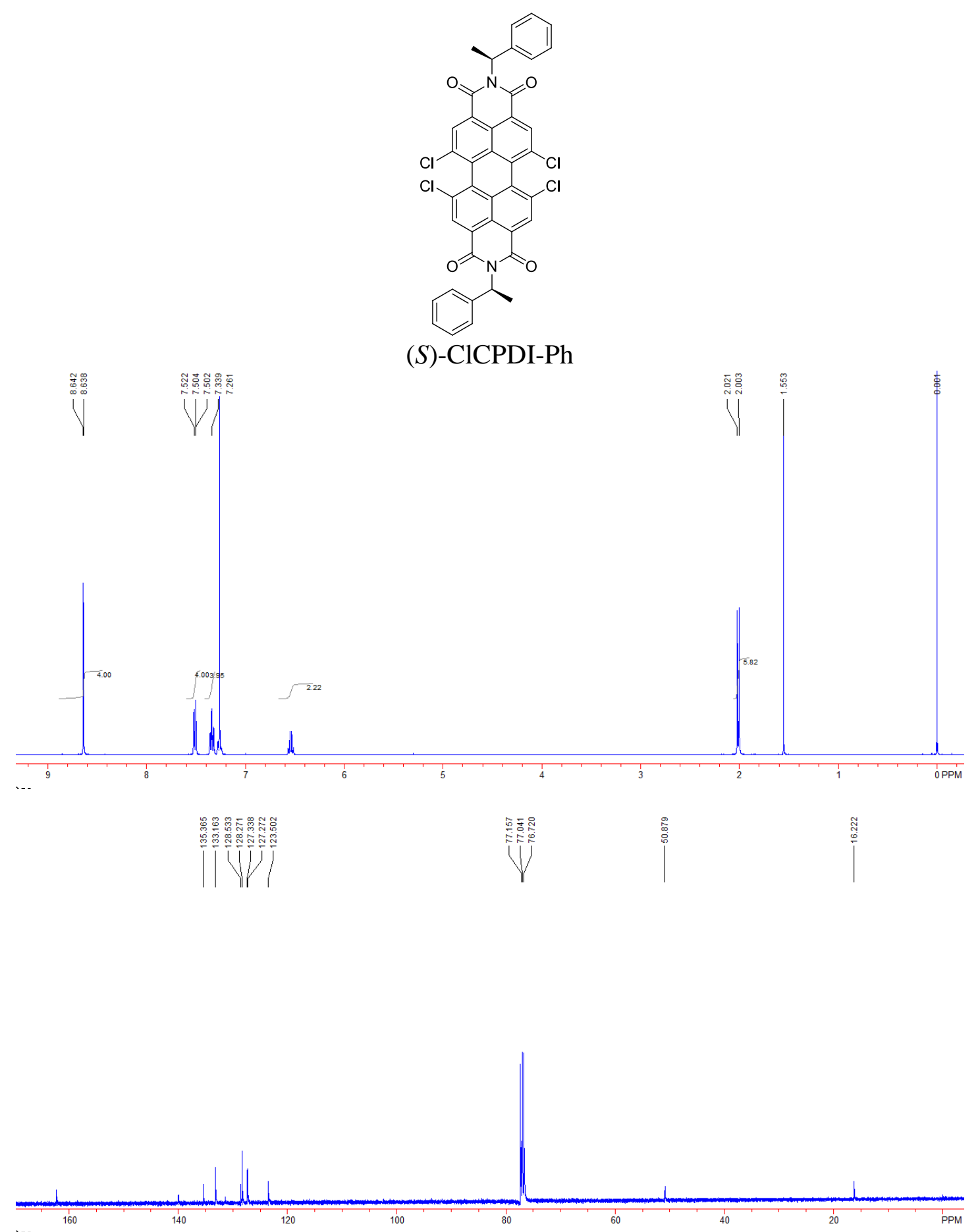

${ }^{1} \mathrm{H}$ NMR (400 MHz, $\mathrm{CDCl}_{3}$ ): $\delta 8.64$ (s, $\left.2 \mathrm{H}\right), 8.64$ (s, $\left.2 \mathrm{H}\right), 7.49-7.54$ (m, $\left.4 \mathrm{H}\right)$, 7.30-7.37 (m, 4 $\mathrm{H}), 7.22-7.30(\mathrm{~m}, 2 \mathrm{H}), 6.54(\mathrm{q}, J=7.2 \mathrm{~Hz}, 2 \mathrm{H}), 2.01(\mathrm{~d}, J=7.2 \mathrm{~Hz}, 6 \mathrm{H}) \mathrm{ppm} .{ }^{13} \mathrm{C}$ NMR $(100$ $\left.\mathrm{MHz}, \mathrm{CDCl}_{3}\right): \delta 162.3,139.3,135.4,133.2,128.5,128.3,127.3,127.3,123.5,50.9,16.2$ ppm. HRMS $(\mathrm{m} / \mathrm{z}):\left[\mathrm{M}^{+}\right]$calcd for 734.0334, found: 735.0409 . 

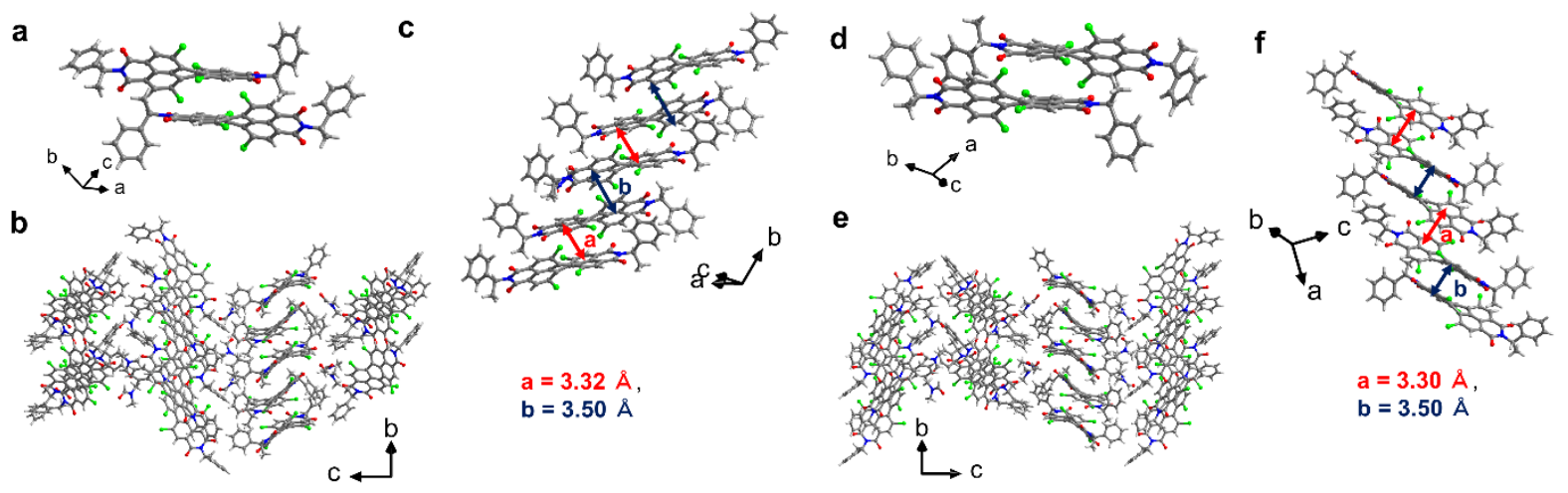

Figure S1. Crystal structure of (R)-ClCPDI-Ph-DMF and (S)-ClCPDI-Ph-DMF; a) asymmetric unit without DMF molecules, b) $a$-axis projection, c) drawing of $\pi-\pi$ stacking for $(R)$-form, d) asymmetric unit without DMF molecules, e) $a$-axis projection, f) drawing of $\pi-\pi$ stacking for (S)-form, Colour code: C: grey, $\mathrm{H}$ : white, $\mathrm{N}$ : blue, $\mathrm{O}$ : red, $\mathrm{Cl}$ : green.
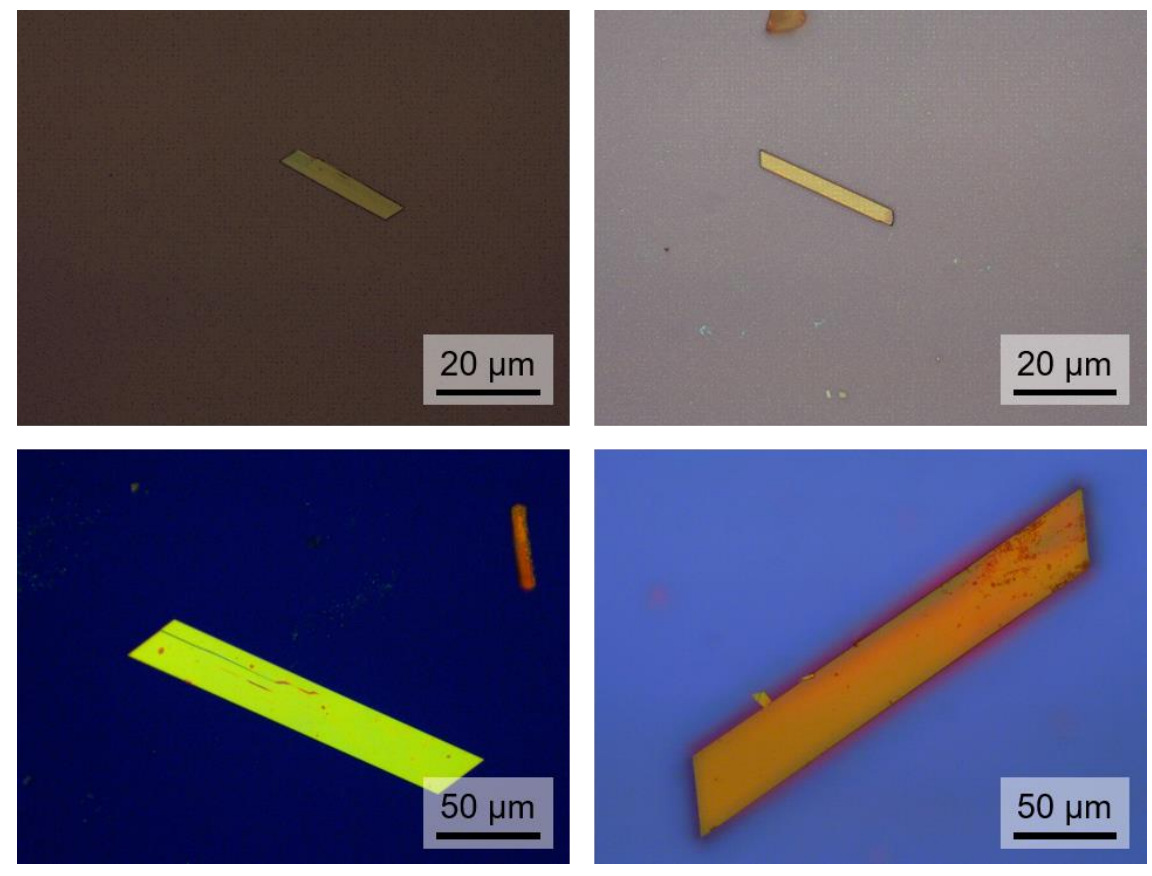

Figure S2. Optical images of ClCPDI-Ph-CF single crystals. 

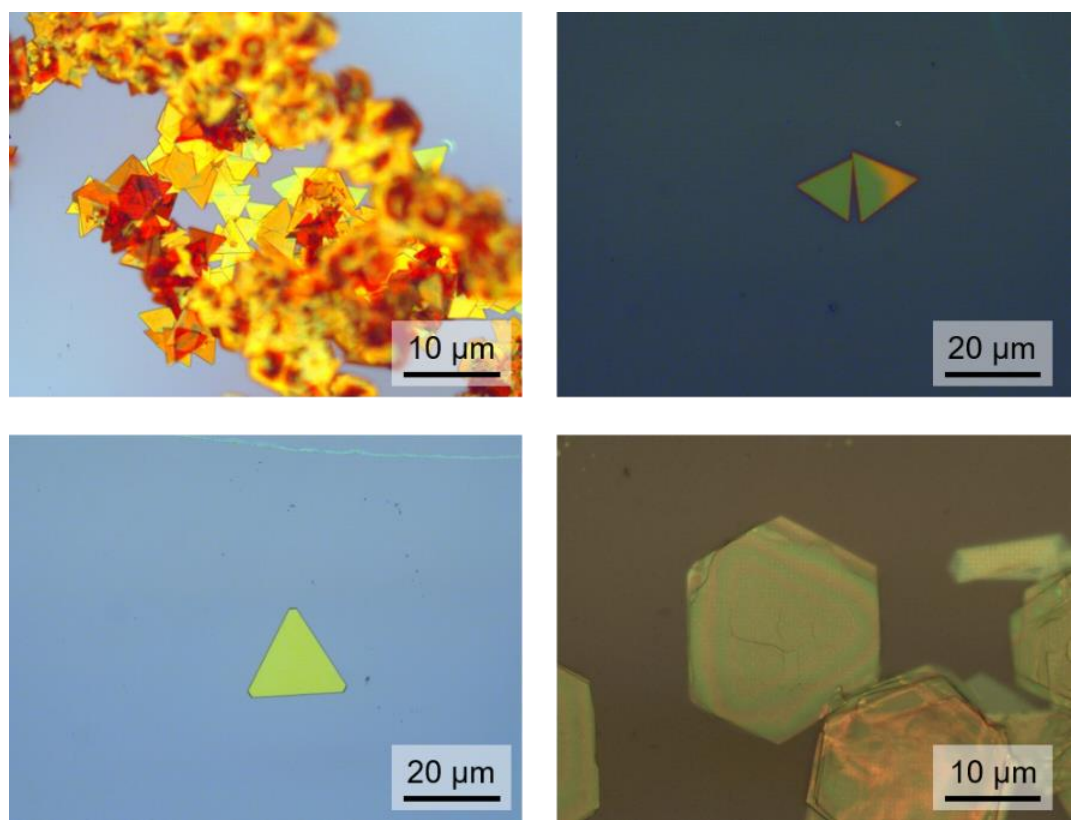

Figure S3. Optical images of ClCPDI-Ph-DMF single crystals.
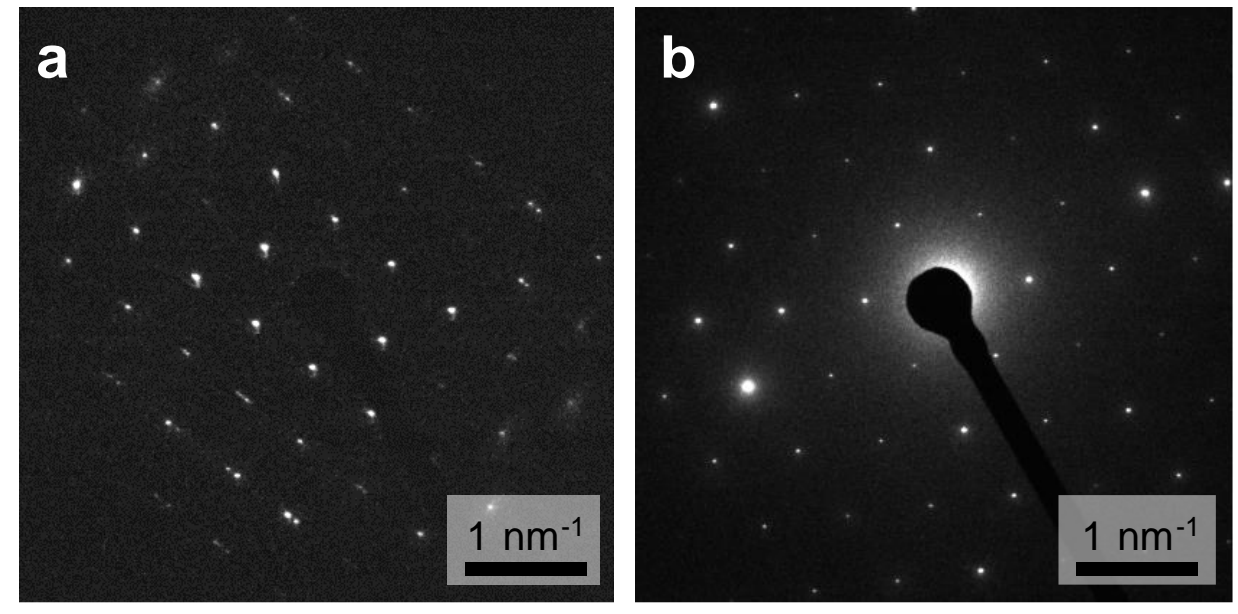

Figure S4. SAED pattern of (a) ClCPDI-Ph-CF and (b) ClCPDI-Ph-DMF single crystals obtained from TEM analysis. 


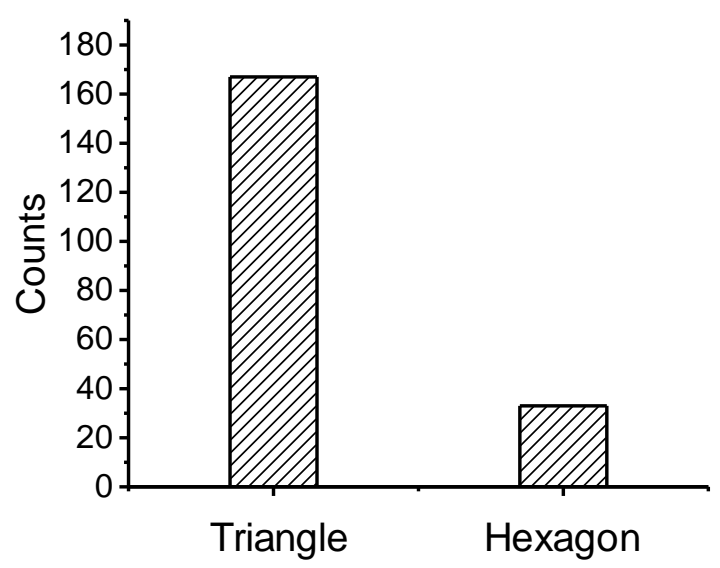

Figure S5. Counts of the number of triangular and hexagonal crystals in self-assembly of ClCPDI-Ph-DMF.
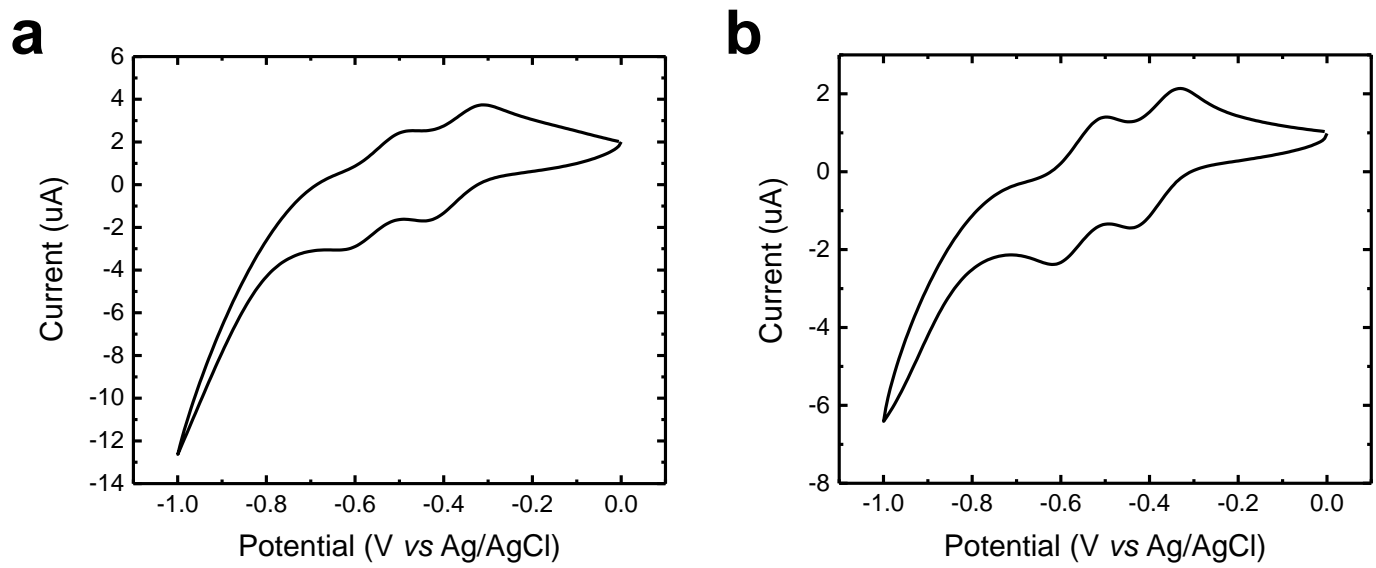

Figure S6. Cyclic voltammetric results of a) (S)-ClCPDI-Ph and b) $(R)$-ClCPDI-Ph chloroform solution $\left(1.0 \times 10^{-4} \mathrm{M}\right)$. 
a

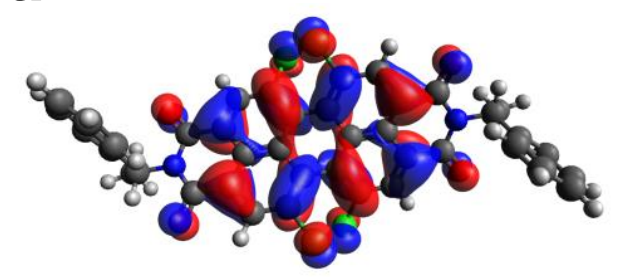

LUMO

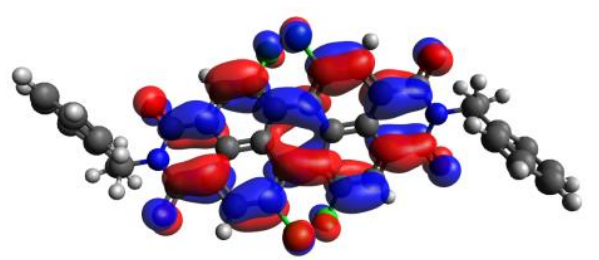

b

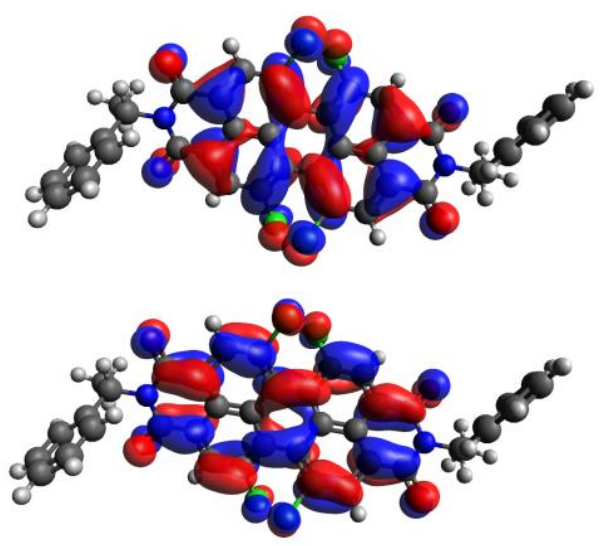

Figure S7. DFT calculation result of ClCPDI-Ph monomer. LUMO and HOMO electron distributions of a) (R)-ClCPDI-Ph and b) (S)-ClCPDI-Ph obtained by DFT calculations. Color scheme is same as Figure 4.

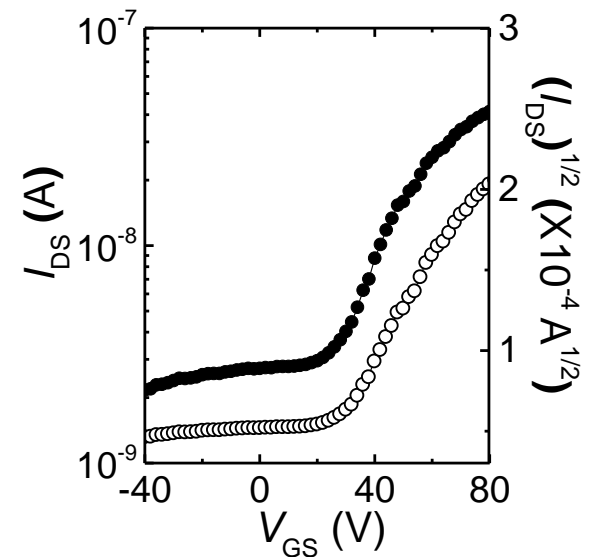

Figure S8. $I_{\mathrm{DS}}-V_{\mathrm{GS}}$ characteristics of $(R)$-ClCPDI-Ph-DMF single crystals in dark and nitrogen conditions. 


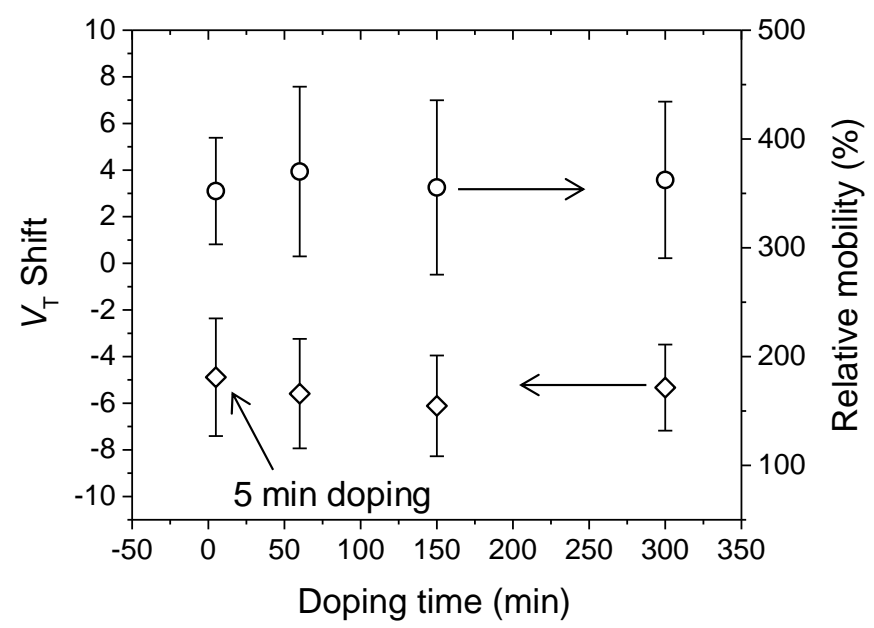

Figure S9. Threshold voltage shift and relative mobility plots of ClCPDI-Ph-CF based OFETs after hydrazine gas doping depending on the doping time.

a

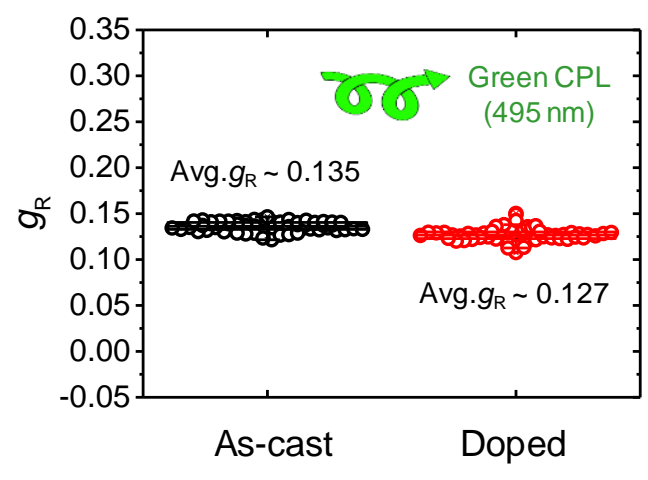

b

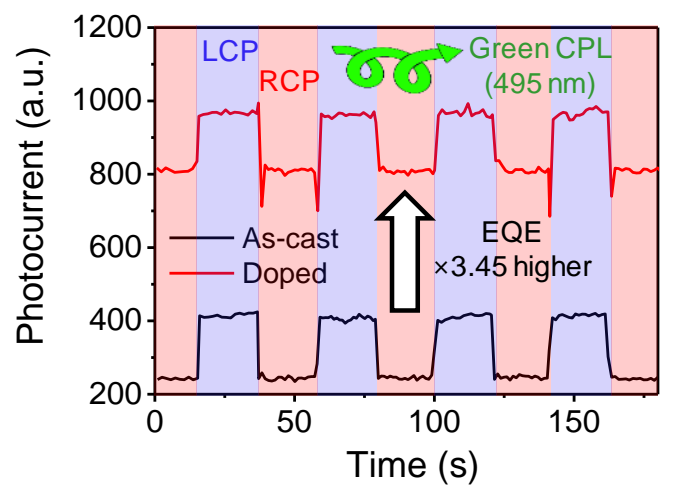

Figure S10. a) Average $g_{\mathrm{R}}$ of an identical device under several cycles of LCPL/RCPL irradiation $(\lambda=495 \mathrm{~nm})$ before and after hydrazine doping. b) Real-time current signals of $(S)$ ClCPDI-Ph-CF SC-OPTs under several cycles of LCPL/RCPL irradiation $(\lambda=495 \mathrm{~nm})$ before and after hydrazine doping. 


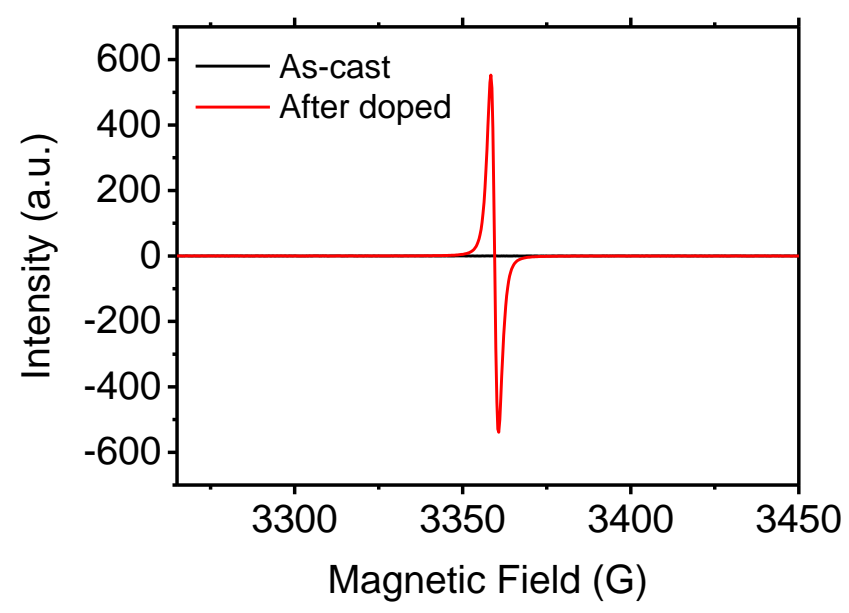

Figure S11. ESR spectra of $(R)$-ClCPDI-Ph-CF single crystals before and after hydrazine doping.

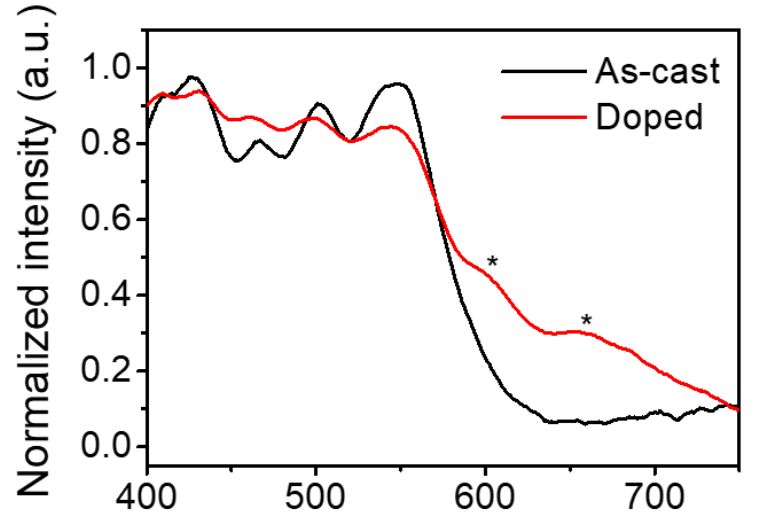

Figure S12. UV-vis spectra of $(R)$-ClCPDI-Ph-CF single crystals before and after hydrazine doping. 

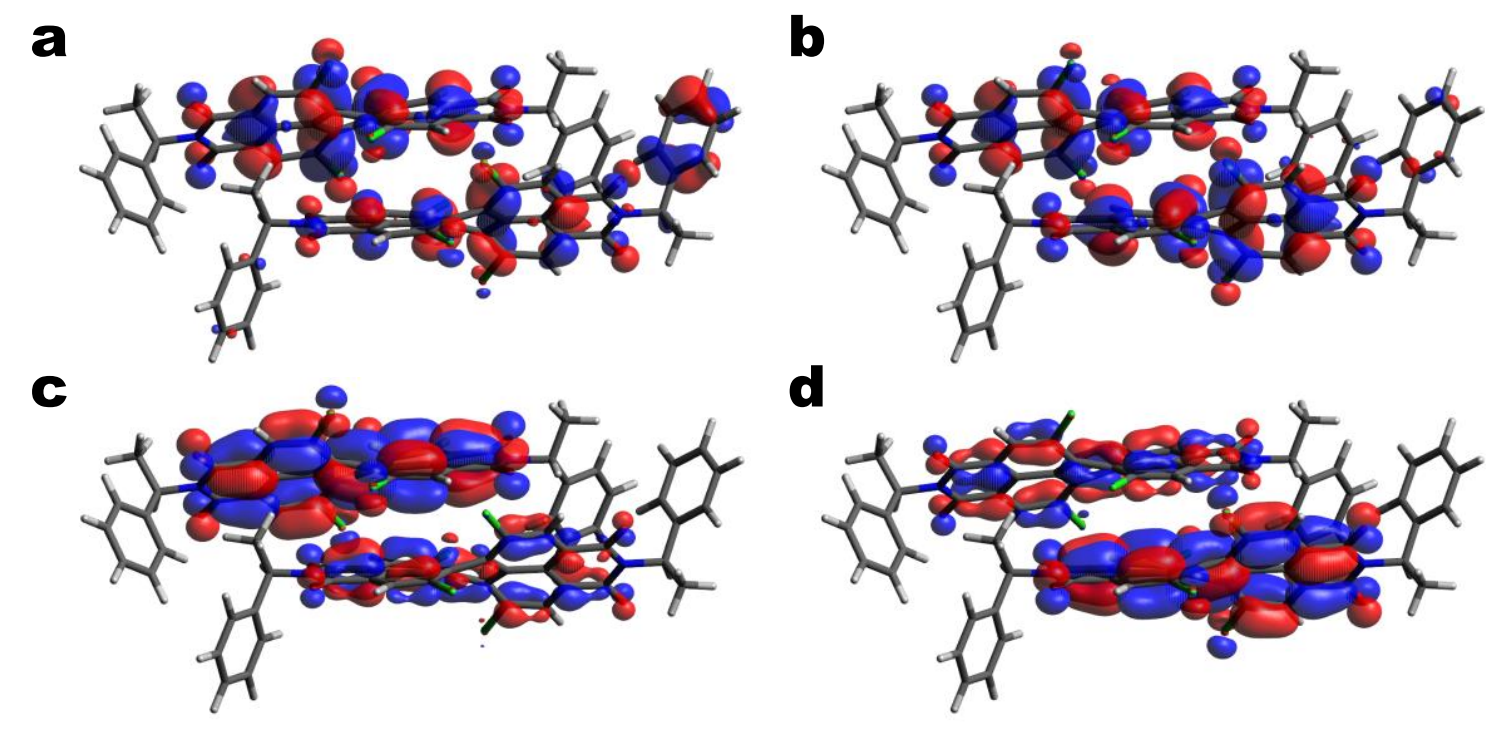

Figure S13. Molecular orbitals of two stacked (R)-ClCPDI-Ph. a) HOMO-1, b) HOMO, c) LUMO, d) LUMO+1. Color scheme is same as Figure 4. 
Table S1. Energy level of enantiomeric ClCPDI-Ph from CV measurement and DFT calculation.

\begin{tabular}{ccccc}
\hline \multirow{2}{*}{ Material } & \multirow{2}{*}{ Chirality } & Orbital & \multicolumn{2}{c}{ Energy level (eV) } \\
& & & CV & DFT \\
\hline \multirow{3}{*}{ ClCPDI-Ph } & $(S)$ & LUMO & -4.06 & -3.89 \\
& & HOMO & -6.32 & -6.46 \\
& $(R)$ & LUMO & -4.05 & -3.89 \\
& & HOMO & -6.31 & -6.46 \\
\hline
\end{tabular}

Table S2. Optoelectronic characteristics of $(R)$-ClCPDI-Ph-CF single crystals based OPTs.

\begin{tabular}{cccccc}
\hline Material & Doping & $R^{a}\left(\mathrm{~A} \mathrm{~W}^{-1}\right)$ & $P^{a}$ & $\mathrm{EQE}^{\mathrm{a}}(\%)$ & $\begin{array}{c}D^{* \mathrm{a}} \\
\left(\mathrm{cmHz}^{1 / 2} \mathrm{~W}^{-1}\right)\end{array}$ \\
\hline $\begin{array}{c}\text { ClCPDI-Ph-CF } \\
\text { single crystals }\end{array}$ & As cast & 187 & $5.7 \times 10^{5}$ & $5.0 \times 10^{4}$ & $1.3 \times 10^{15}$ \\
& Doped & 1129 & $1.7 \times 10^{7}$ & $3.0 \times 10^{5}$ & $2.2 \times 10^{16}$ \\
\hline
\end{tabular}

${ }^{a}$ Maximum values under monochromic light illumination $\left(\lambda=460 \mathrm{~nm}, 50 \mu \mathrm{W} \mathrm{cm}{ }^{-2}\right)$.

Table S3. Doping effect of hydrazine on electron affinity of $(R)$-ClCPDI-Ph molecule.

\begin{tabular}{ccccccc}
\hline Material & & $E_{\text {neutral }}(\mathrm{Ha})$ & $E_{\text {anion }}(\mathrm{Ha})$ & $E_{\text {cation }}(\mathrm{Ha})$ & $\mathrm{EA}(\mathrm{eV})$ & $\mathrm{IP}(\mathrm{eV})$ \\
\hline \multirow{3}{*}{$(R)$-ClCPDI-Ph } & Bare & -3981.288 & -3981.428 & -3981.049 & 3.789 & 6.499 \\
& Doped & -4099.059 & -4099.199 & -4098.844 & 3.827 & 5.843 \\
\hline
\end{tabular}

